\title{
El puzle de las teorías de las proteínas funcionales: ¿qué tiene en común el ibuprofeno con el envenenamiento por monóxido de carbono? Una respuesta filosófica a un problema científico*
}

The Puzzle of the Theories of Functional Proteins: What has in Common with Ibuprofen Carbon Monoxide Poisoning? A Philosophical Answer to a Scientific Problem

Lucía Federico $^{\dagger \neq}$

\section{Resumen}

En el presente artículo se muestra mediante un análisis epistemológico que dos fenómenos biológicos paradigmáticos no son explicados por las teorías usualmente empleadas en el área. Del análisis se predice por tanto que en la bibliografía hay una alternativa superadora implícita.

Palabras clave: proteína - teoría Monod-Wyman-Changeux - reducción teórica - inconmensurabilidad

\begin{abstract}
Absract
In this article it is shown by an epistemological analysis that two paradigmatic biological phenomena are not explained by the theories usually employed in the area. From such analysis it is therefore predicted that in the literature there implicitly exists a proper alternative.
\end{abstract}

Keywords: protein - Monod-Wyman-Changeux theory - theoretical reduction - incommensurability

* Recibido: 27 de Marzo de 2016. Aceptado con revisiones: 5 de Diciembre de 2016.

+ Centro de Estudios de Filosofía e Historia de la Ciencia (CEFHIC), Universidad Nacional de Quilmes (UNQ). Para contactar al autor, por favor, escribir a: luciafed@hotmail.com.

* Agradezco a los evaluadores por sus sugerencias, que ayudaron a mejorar la calidad del presente artículo, y a la Médica Carolina Vásquez por su amistad e incalculable ayuda a la hora de rastrear material sobre alosterismo. Este trabajo ha sido realizado con la ayuda de los proyectos de investigación PICT-2012-2662 y PICT-2014-1741 (ANPCyT, Argentina).

Metatheoria 8(1)(2017): 117-126. ISSN 1853-2322.

(C) Editorial de la Universidad Nacional de Tres de Febrero. Publicado en la República Argentina. 


\section{Introducción}

Difícilmente nos encontremos en estos tiempos con alguien que no utilice ibuprofeno para aplacar algún dolor. ¿Quién no escuchó alguna vez noticias de decesos hogareños, producto del envenenamiento con monóxido de carbono? Y si bien estos sucesos resultan familiares, el lector promedio no los encuentra vinculados. Más notorio es que aún para el biólogo tampoco sea una tarea sencilla. Qua filósofos de la ciencia creemos posible precisar la desazón que sufre el científico a la hora de vincularlos y demostrar que pese a la vicisitud es una tarea factible. Para ello usaremos el instrumental de elucidación que nos brinda la metateoría estructuralista de las teorías científicas. ${ }^{1}$

Antes de avanzar conviene hacer una primera presentación de los casos en lenguaje científico.

El ibuprofeno es un fármaco que tiene como diana una enzima que poseen algunas células de nuestro cuerpo. Las enzimas son las macromoléculas que catalizan las reacciones químicas dentro (y a veces fuera) de las células. En los procesos de inflamación y en el fisiológico de protección de la mucosa gastrointestinal actúa la prostaglandina. El ibuprofeno tiene su acción farmacológica sobre la Ciclooxigenasa (COX), la enzima clave en la producción de prostaglandina a partir del ácido araquidónico. El fármaco inhibe su producción al competir por el centro catalítico de la enzima, lugar donde ocurre la oxidación química del ácido araquidónico. Así consigue su efecto de disminuir el dolor e inflamación, pero también, como efecto secundario, produce daños en la pared gastrointestinal.

Por otro lado, entre los causales de muerte doméstica más comunes (un 50\%) se encuentra la intoxicación por monóxido de carbono (ICO), producto de una combustión incompleta por falta de oxígeno. La hemoglobina, macromolécula encargada del trasporte de oxígeno a los distintos tejidos del cuerpo, es capaz de ligar el monóxido de la misma forma que lo hace con el oxígeno. Ambas moléculas son semejantes, pero la afinidad del CO por los centros de unión de la hemoglobina es mayor que la del oxígeno. En presencia de ambas moléculas el CO gana la competencia y la hemoglobina termina trasportando una sustancia tóxica a los tejidos.

De esta primera aproximación concluimos que en ambos casos se encuentra implicada una proteína cuya función normal se ve afectada por sustancias que compiten con sus habituales sustratos (las sustancias con la que cumplen su acción fisiológica). Con esta caracterización un lector con conocimiento en biología señalará que actualmente existe un conjunto de "teorías" (también llamados en el ámbito científico "modelos" o "ecuaciones") que dan cuenta de este tipo de patrones de actividad.

En otro plano conceptual de análisis, el filósofo advertirá que este es un primer paso para hallar el vínculo entre ambos sucesos, pues el científico percibe que son fenómenos explicados por teorías de una misma área. Nuestra tesis es que, aunque heterogéneos, son casos de aplicación de una misma teoría y que son fenómenos de un mismo "tipo" (en el sentido de "ejemplar” kuhniano).

Siguiendo la intuición del científico, en un escaneo pormenorizado de los libros de textos más usados en el área (lugar donde se presentan las teorías consensuadas por la comunidad de expertos) encontramos dos candidatos habitualmente utilizados para dar cuenta de esta clase de fenómenos biológicos: la teoría michaeliana y el llamado Modelo Alostérico Concertado. Cabe agregar aquí que existe un cierto "entendimiento tácito" en el área de que esta última al ser una propuesta "más compleja" (y explicar más sistemas) que la michaeliana, resulta ser una propuesta "superadora".

A continuación, presentaremos la reconstrucción informal del Modelo Alostérico, pues ya se cuenta con una reconstrucción en la literatura estructuralista, con el fin de allanar la tarea que aquí nos ocupa.

\footnotetext{
${ }^{1}$ Véase Balzer, Moulines y Sneed (1987).
} 


\section{El modelo alostérico}

En los años '60 se descubrió que la actividad de muchas enzimas presentaba una curva sigmoidea o "de cooperatividad”. Esto resultó sorprendente, pues las enzimas conocidas hasta ese momento exhibían una curva hiperbólica. Pero hubo que esperar hasta 1965 para contar con una propuesta que diera cuenta del efecto cooperativo. Los autores de tal hazaña fueron Jacques Monod, Jeffries Wyman y JeanPierre Changeux, desarrolladores del "modelo alostérico concertado" o "MWC", por sus autores. El mismo presentaba un mecanismo molecular alostérico para explicar correlaciones entre el cambio en la actividad biológica y la presencia de diferentes ligandos en proteínas cooperativas. Lo resonante del modelo se debió probablemente al prestigio del Premio Nobel Monod y a lo audaz que parecía este mecanismo. ${ }^{2}$

A continuación, expondremos sucintamente la reconstrucción de la teoría, que puede verse completa en Alleva, Díez y Federico (2012).

La novedad del modelo se debió al planteo, como conceptos explicativos, de dos "estados conformacionales" para una misma proteína. Cada estado conformacional presenta un tipo de estructura tridimensional y de actividad biológica: el estado "tenso" (T) con baja actividad y el estado "relajado" (R) con alta actividad. Cada estado conformacional se une, con una afinidad particular, a cada tipo de ligando. El ligando es toda sustancia a la cual la proteína se une, sea su sustrato u otra sustancia.

Podemos decir entonces que los componentes teóricos de la teoría, aquellos conceptos nuevos introducidos por ésta (Hempel 1958) son: los estados conformacionales representados por $\{\tau, \mathrm{r}\}$ respectivamente; el estado conformacional en que se encuentra la proteína en un momento particular representado por $\zeta$ y las constantes de afinidad para cada estado conformacional representadas por $\kappa \Psi_{\tau}$ y $\kappa{ }_{r} r$ respectivamente.

Los tipos de sistemas biológicos que la teoría pretende explicar, representados como modelos de datos, son estructuras ordenadas del tipo: $\langle O, S, A, I, T, \rho, \beta, \sigma,|\Psi t|, \delta\rangle$ donde $O$ representa las proteínas de una población (llamadas oligoméricas por tener más de una subunidad); $S, A, I$ representan los ligandos (sustrato, activador e inhibidor); $T$ representa el tiempo; $\rho$ representa la cantidad de subunidades (o protómeros) de las proteínas de la población (que es igual al número de sitios de unión para cada ligando y es constante); $\beta$ representa los estados de unión de las enzimas (unida, no unida, etc.); $\sigma$ representa la evolución de los estados de unión de las proteínas en el tiempo; $\left|\Psi_{t}\right|$ es la cantidad de ligando unido a las enzimas y $\delta$ la actividad biológica.

Agregando éstos a los anteriores, los componentes de la teoría, en una estructura ordenada, son: $\left\langle O, S, A, I, T,\{\tau, r\}, \rho, \sigma, \beta,\left|\Psi_{t}\right|, \delta, \zeta, \kappa_{S}, \kappa_{A}, \kappa_{I}\right\rangle$, es decir, los dominios (base ontológica de la teoría) y sus relaciones y funciones.

Nuestra reconstrucción muestra que la teoría presenta tres leyes fundamentales que deben cumplirse en las aplicaciones, si bien en la bibliografía no se encuentran explicitadas. La primera asume que en ausencia de ligando los estados $\mathrm{T}$ y $\mathrm{R}$ de una población están en equilibrio: el total de proteínas en estado $\mathrm{T}$ y el total en estado $\mathrm{R}$ son constantes. El valor de la relación entre los estados es conocido como "constante alostérica" $\left(\mathfrak{l}_{0}\right)$. La segunda restricción asume que sólo las formas no unidas a ligando, en cualquiera de los estados, pueden cambiar de conformación. Ese cambio fue denominado "transición alostérica" (de todas las subunidades juntas y por eso "concertado"). Una vez unida la proteína a un ligando queda "fijada" en una conformación no pudiendo producirse una nueva transición alostérica. Por último, la tercera ley vincula el cambio conformacional con la actividad biológica de esta manera:

$$
\delta_{(t)}=\frac{\left[l_{0 t} \cdot\left(1+\varepsilon_{I \tau t}\right) \rho o /\left(1+\varepsilon_{A r t}\right) \rho o\right] \cdot \varepsilon_{s t t} \cdot\left(1+\varepsilon_{s t t}\right) \rho o-1+\varepsilon_{s t t} \cdot\left(1+\varepsilon_{s r t}\right) \rho o-1}{\left[l_{0 t} \cdot\left(1+\varepsilon_{I \tau t}\right) \rho o /\left(1+\varepsilon_{A r t}\right) \rho o\right] \cdot\left(1+\varepsilon_{s t t}\right) \rho o+\left(1+\varepsilon_{s r t}\right) \rho o}
$$

\footnotetext{
${ }^{2}$ De los modelos alostéricos aceptados, los más populares son el de Hill y el MWC (Kesse \& Ben-Tal 2011, p. 168), pero el último es el que presentaría un mecanismo explicativo.
} 
Def. Aux.: $\varepsilon \Psi_{t}=\left|\Psi_{t}\right| / \kappa_{\Psi}$ (es la cantidad de ligando "normalizado" en un estado conformacional).

Esta ley establece que el grado de actividad coincide "cualitativamente" con la proporción de proteínas unidas (en ambos estados conformacionales) sobre el total de uniones posibles en la población.

Aunque estas leyes sean abstractas y de amplio alcance (subscriptas en el marco de interés de la teoría) son susceptibles de sufrir una serie de modificaciones, introduciendo restricciones que limiten su generalidad, de manera de alcanzar las distintas "formas" que adoptan para explicar los sistemas empíricos que pretende dar cuenta la teoría. Estas leyes de carácter restricto llevan el nombre de "leyes especiales" (Balzer, Moulines \& Sneed 1987). Para el estructuralismo, las teorías son un conjunto de elementos teóricos, cada uno con su ley de mayor o menor grado de generalidad, vinculados entre si por la relación de "especialización" (a medida que se introducen las restricciones). El elemento teórico más general se encuentra en la cúspide mientras que los más especializados están en la base. Este ensamble forma una "red teórica", más afín a la noción de "matriz disciplinar" kuhniana que al concepto clásico de teoría.

Entonces, las leyes adquieren distintas formas según los sistemas a los que se aplica la teoría. Entre estos encontramos aquellos donde sólo hay presencia de sustrato ("homotrópicos"), aquellos donde actúan sustrato y moduladores activadores y/o inhibidores ("heterotrópicos"), y aquellos donde las enzimas son o se comportan como michaelianas. Esta afirmación de los autores (Monod, Wyman \& Changeux 1965), y repetida en la bibliografía, es la que genera la presunción de que ésta es una teoría "superadora" de la michaeliana.

Retomemos al ibuprofeno y al monóxido de carbono. La COX posee dos subunidades y, como la hemoglobina, es un oligómero. Ambos sistemas parecen ser explicados por MWC. Pero el ibuprofeno y el monóxido son dos inhibidores, entonces avancemos sobre las especializaciones dónde se tienen en cuenta la acción de los moduladores.

Los moduladores son sustancias que modifican el patrón de actividad, acelerándolo o inhibiéndolo. En esta teoría ambos ligandos actúan ejerciendo su acción mediante su unión en un sitio específico ("alostérico") y a un estado conformacional de la proteína. Cada subunidad, además del sitio catalítico, presenta en general un sitio de unión para cada modulador. El estado R tiene alta afinidad por el activador mientras que el estado $\mathrm{T}$ lo tiene por el inhibidor.

Ahora bien, la asunción de estos supuestos, útiles para explicar la inhibición "por producto final" del metabolismo celular, nos presenta un fuerte impedimento al contemplar nuestros ejemplos como aplicaciones de la teoría: no incluye otra clase de inhibición que aquella producto de la unión a un sitio específico distinto al catalítico, a diferencia de nuestros dos inhibidores que compiten por unirse al mismo sitio que el sustrato. Y con ello se genera un problema. Si el ibuprofeno y el monóxido compiten por la unión a tal sitio, lo harán al igual que el sustrato, predominantemente cuando la proteína esté en estado R, con el centro catalítico "expuesto". Pero el inhibidor modulador lo hace uniéndose a una región expuesta en el estado $\mathrm{T}$, estabilizando la proteína en su forma poco activa.

Existe un dilema adicional: si la tercera ley de la teoría alostérica no contempla inhibidores competitivos (ni tampoco los no competitivos que actúan uniéndose de forma inespecífica) ¿qué "sistemas michaelianos", que sí contemplan sistemas con dichos inhibidores, son los que explica MWC?

Gracias a nuestra reconstrucción podemos precisar que MWC sólo captura sistemas donde las enzimas interaccionan únicamente con sustrato y lo hace asumiendo que: la cantidad inicial de proteína en estado $\mathrm{T}$ es despreciable frente a la cantidad en estado $\mathrm{R}\left(l_{0 t}=0\right)$, las proteínas están formadas por una subunidad (un solo sitio de unión) y las constantes de afinidad de los distintos estados conformacionales por el sustrato son iguales $\left(\varepsilon_{s r t}=\varepsilon_{s \pi}\right)$. Bajo estas restricciones la ley toma una forma especializada equivalente a la "ecuación michaeliana": $\delta_{(t)}=\varepsilon_{s t} / 1+\varepsilon_{s t}$.

Lo interesante es que la presunción de que esta teoría es "superadora" de la michaeliana no parece darse (aunque falta demostrarlo). Probemos entonces con la teoría michaeliana, la otra teoría más usada en el área. 
En la bibliografía estructuralista no se cuenta con una propuesta reconstructiva previa así que, la aquí presentada, es su primera reconstrucción modelo-teórica, si bien no la desarrollaremos con el detalle formal que quisiéramos por una cuestión de espacio.

\section{La cinética michaeliana}

Los estudios acerca de las curvas de actividad de las enzimas llevaron a la conformación de una nueva subdisciplina: la enzimología. La propuesta desarrollada por Leonor Michaelis y Maud Menten en 1913 y cristalizada con los aportes de George E. Briggs y John B.S. Haldane en 1925, describe cómo varía la velocidad de catálisis a medida que varía la cantidad de ligando.

En una curva de actividad, a medida que el sustrato aumenta, la velocidad de reacción aumenta hasta llegar a una meseta, la velocidad máxima, momento en que todas las enzimas se encuentran "ocupadas" por sustrato. Estas curvas constituyen los tipos de fenómenos biológicos que la teoría pretende explicar, y pueden ser representados como un modelo de datos por los siguientes componentes: $\langle E, S, I, T, \beta, \sigma, \delta,|\Psi t|\rangle . E$ representa a las proteínas funcionales (enzimas en este caso) ${ }^{3}$ de una población; $S$ e $I$ representan los ligandos (sustratos e inhibidores respectivamente); $\beta$ representa los estados de unión de las enzimas; $\sigma$ representa la evolución de los estados de unión de las enzimas en el tiempo (representado por $T$ ); $\left|\Psi_{t}\right|$ es la cantidad de ligando unido a las enzimas y $\delta$ la actividad biológica.

Como señalamos, esta teoría da cuenta de la actividad de las proteínas incluso en presencia de inhibidores por lo cual parece ser una buena candidata para explicar la acción del ibuprofeno y del envenenamiento por monóxido de carbono. Aunque el COX sea una enzima y la hemoglobina un transportador, el usuario de la teoría en principio no hace diferencia.

Las proteínas funcionales poseen una afinidad particularmente alta por su sustrato y poca por otras sustancias, por lo cual se las considera moléculas "específicas". Este valor de la afinidad es representado mediante la "constante de afinidad": $\kappa_{S}, \kappa_{I}$ para cada ligando respectivamente y es el "componente explicativo" (el término teórico) de la teoría (Díez 2002).

Recapitulando, podemos decir que la totalidad de los componentes de la teoría, presentados como conjunto ordenado, son: $\left\langle E, S, I, T, \beta, \sigma, \delta,\left|\Psi_{t}\right|, \kappa_{S}, \kappa_{I}\right\rangle$.

Según nuestra propuesta, en la teoría michaeliana la ley fundamental o generalización simbólica ${ }^{4}$ conecta la actividad biológica con el grado de afinidad de las enzimas a los ligandos, sustrato e inhibidores. Esta regularidad nómica establece que a cada momento la actividad coincide "cuantitativamente" con la proporción de enzimas de la población cuyos sitios catalíticos se encuentran ocupados por ligando, sobre el total de uniones posibles en la población. Formalmente:

$$
\delta(t)=\varepsilon_{s t} /\left(1+\mathcal{E}_{i t}{ }^{\prime}\right)+\varepsilon_{s t}\left(1+\mathcal{E}_{i}^{\prime \prime}\right) \text {, donde Def. Aux.: }{ }^{5} \mathcal{E}_{t}=\left|\Psi_{t}\right| / \kappa_{\Psi}(\text { es la cantidad de ligando "normalizado"). }
$$

Igual que en lo presentado en la reconstrucción anterior, la ley adquiere distintas formas según los sistemas a los que se aplica la teoría. Entonces, si en los sistemas físicos (o aplicaciones intencionales de la teoría) sólo hay presencia de sustrato y ningún otro ligando (primera restricción), la ley general adquiere una forma equivalente a la presentada en los libros de texto como "ecuación de MichaelisMenten": $\delta_{(t)}=\left|\Psi_{s t}\right| / \kappa_{s}+\left|\Psi_{s t}\right|$ donde $\kappa_{s}$ es la "constante michaeliana" $(K M)$, parámetro de afinidad de la enzima por su sustrato.

Pero si hay además presencia de inhibidor, la ley general adquiere dos formas distintas, en función de cómo actúe ese inhibidor.

Si la sustancia compite con el sustrato por unirse al sitio catalítico, al interferir con la unión el valor de $K M$ aparenta ser de menor afinidad (y es la nueva restricción introducida). Esto genera que la

\footnotetext{
3 Actualmente también se aplica a la mioglobina, otro transportador, y a algunos receptores.

4 Debemos mencionar que la expresión matemática de la ley no aparece en la bibliografía científica explicitada como tal.

5 ' $\varepsilon \Psi$ ' se corresponde con la cantidad de uniones de ligando $\Psi$ a la enzima $e$ en el momento t, dividido la constante de disociación $\kappa$ para el ligando correspondiente.
} 
velocidad catalítica disminuya pero una cantidad muy grande de sustrato permite restablecer la velocidad. La forma que adquiere la ley en estos sistemas llamados de "inhibición competitiva" es: $\delta(t)=\varepsilon_{s t} / \varepsilon_{s t}+\left(1+\varepsilon_{i t}\right)$.

Si la sustancia se une al mismo tiempo que el sustrato en otro sitio inespecífico, el valor de $K M$ no parece modificarse (otra restricción) y el incremento de sustrato no contrarresta la inhibición. La forma que toma la ley para estos sistemas llamados de "inhibición no-competitiva" es: $\delta(t)=\varepsilon_{s t} / 1+\varepsilon_{s t}\left(1+\varepsilon_{i}{ }^{\prime \prime}\right)$.

Volvamos a nuestros casos. Como en estos sistemas ocurre una inhibición competitiva consideramos entonces que la teoría michaeliana podría ser una buena candidata para explicar la alteración de las funciones biológicas descriptas, bajo la ley especial de "inhibición competitiva": estos sistemas parecen ser aplicaciones intencionales de dicha teoría.

Sin embargo, la teoría fue desarrollada para un tipo particular de proteínas, pues asume que cada enzima sólo tiene un sitio de unión o "catalítico". Entonces volvamos a examinar con más detalle cómo son nuestras proteínas.

La hemoglobina es un tetrámero formado por cuatro subunidades unidas entre sí. Cada subunidad posee un grupo "hemo", una estructura anular que contiene un átomo de hierro, capaz de unir un oxígeno. Además, la unión de un oxígeno incrementa la probabilidad de unión de otro oxígeno en la subunidad vecina, el fenómeno llamado "cooperatividad".

Lamentablemente la función matemática de la ley fundamental de la teoría michaeliana contempla sólo una unión por molécula, e ignora el efecto cooperativo. Esto descarta al sistema hemoglobina como aplicación de la teoría, ¿y la ciclooxigenasa?

La estructura de COX no es habitual en la bibliografía, pero encontramos su descripción en artículos especializados. Se trata de un dímero que, en cada subunidad, posee un sitio activo ciclooxigenasa y otro peroxidasa (que activa el grupo hemo que participa en la reacción de la ciclooxigenasa). Entonces, si bien es una enzima y no un transportador, presenta semejanzas con la hemoglobina: posee más de una subunidad, una estructura simétrica, contiene grupos hemo y presenta cooperatividad (Worldwide Protein Data Bank 2016, Zou et al. 2012). No nos queda más que también descartar el sistema COX como aplicación intencional de la teoría.

Lo cierto es que ni una ni otra teoría explica la acción del ibuprofeno y del monóxido. Esto resulta curioso tratándose de fenómenos harto conocidos.

En este punto es donde quizás el lector con conocimiento científico quede un tanto alarmado, pues, aunque haga uso de su memoria, caerá en la pregunta del lector lego: ¿qué teoría los explica? Es el momento de buscar una teoría alternativa.

\section{El auxilio filosófico a un problema científico}

Con las dos reconstrucciones presentadas y los casos analizados, diremos que nuestra tesis filosófica es i) que no existe una relación de reducción entre MWC y la teoría michaeliana (o que una "supere" a la otra); ii) que la relación es de inconmensurabilidad parcial; iii) hay una tercera teoría, no explicitada en la bibliografía básica del área, capaz de explicar los fenómenos analizados y pasible de explicitación mediante el instrumental estructuralista.

Para que se cumpla la relación de reducción entre dos teorías (llamemos a una teoría T reducida y a la otra teoría $\mathrm{T}^{*}$ reductora) se deben dar las siguientes condiciones: i) tiene que haber una "correspondencia global" entre los marcos conceptuales de las teorías $\mathrm{T}$ y $\mathrm{T}^{*}$, es decir entre los conjuntos de sus componentes; ii) la correspondencia también se tiene que dar al nivel de las aplicaciones, es decir las aplicaciones intencionales de $\mathrm{T}$ deberán tener un correlato en $\mathrm{T}^{*}$ pero no necesariamente a la inversa; y iii) siempre que una aplicación cumpla las leyes de la teoría reductora $\mathrm{T}^{*}$ (la general y las restricciones específicas impuestas por un sistema particular), entonces en la $T$ reducida el correlato de esa aplicación cumplirá también las leyes de la teoría reducida T (Balzer, Moulines \& Sneed 1987). 
De la presentación de las reconstrucciones y su comparación se concluye que ni (la 3o ley de) la teoría alostérica (que llamaremos TMWC), ni (la ley de) la teoría de Michaelis-Menten (TMM), son factibles de ser vinculadas mediante la relación de reducción, al menos por tres razones: i) entre los componentes (conceptos) de la teoría MWC hay ausencia de ciertos componentes (conceptos) de los que hace uso la teoría MM, por tanto, no hay una correspondencia entre marcos conceptuales; ii) mientras que la teoría alostérica captura la interacción de las proteínas con los ligandos moduladores inhibidores, la teoría michaeliana captura la interacción de las proteínas con los inhibidores no moduladores, no habiendo tampoco una correspondencia de aplicaciones; y iii) la teoría MWC puede dar cuenta de los sistemas "michaelianos" pero sólo aquellos capturados por la "ecuación de MichaelisMenten" (en ausencia de inhibidores) y no de los restantes sistemas de la teoría MM. Así la forma que adoptan las leyes se tornan incompatibles. Por ello rechazamos que entre estas teorías exista una relación de reducción teórica.

En segundo lugar, creemos que la relación que se da entre ellas es de inconmensurabilidad local.

Según Kuhn dos teorías son inconmensurables si sus "estructuras léxicas" no son "homologables", es decir, no reflejan el mismo mundo. Entonces, dos teorías son inconmensurables si sus lenguajes (términos/conceptos) no son "intertraducibles".

La inconmensurabilidad local se da sólo en las áreas donde las dos taxonomías difieren y por ello los problemas de traducción ocurren entre una subclase de términos (relacionados semánticamente) y no con todos los términos de la teoría, donde su significado se preserva de teoría a teoría (Jaramillo 2012). Sin embargo, la no traducibilidad no implica "incomparabilidad", pues de ello no se infiere que los científicos usuarios de una teoría no puedan "comprender" la otra mediante un proceso de interpretación (aprendiendo el lenguaje).

Así Kuhn propone una tesis de la inconmensurabilidad semántica en términos estructurales sin tratamiento formal. Pero relacionando su tesis taxonómica con concepciones semánticas podemos precisar los siguientes aspectos: dos teorías alternativas son "teóricamente inconmensurables" cuando difieren sus "marcos conceptuales" (el conjunto de componentes de las teorías) y esto se da cuando al menos difieren sus conceptos teóricos. A su vez dos teorías son "empíricamente comparables" si hay una correspondencia entre "las bases empíricas" (el conjunto de los modelos de datos) y por tanto entre sus términos no teóricos. Así podría darse la situación de que compartan también algunas aplicaciones intencionales o que no todos los términos con problemas de traducibilidad se identifiquen con los teóricos, sino que también lo hagan con algunos no teóricos (Lorenzano 2012).

La situación planteada aquí se vincula con esto último. Comparando las estructuras que reflejan la base empírica de ambas propuestas, "aquello de lo que hablan las teorías", podemos establecer ciertas conexiones entre ellas (hablan de lo mismo), pero la relación no es de identidad. Si bien la mayoría de los conceptos coinciden (sustrato, tiempo, estado de unión, evolución de los estados de unión, cantidad de ligando unido y actividad biológica), en MWC se quiere dar cuenta de situaciones donde operan ligandos inhibidores que no son los de la teoría michaeliana y viceversa. Además, en la propuesta michaeliana no hay sistemas donde actúen los ligandos activadores que aparecen en algunos sistemas alostéricos. Adicionalmente para caracterizar las proteínas michaelianas, al ser "más sencillas", no es necesario introducir el concepto de subunidad, necesario para la cooperatividad alostérica. Entonces sostendremos la existencia de "una ontología parcialmente en común" entre teorías según sus conjuntos base.

Comparando sus marcos conceptuales notamos que estas estructuras no son homólogas. En parte por lo analizado, en parte porque difieren en sus conceptos explicativos (en Michaelis aparecen constantes de afinidad para cada tipo de ligando, y en MWC aparecen los estados conformacionales, la afinidad para cada tipo de ligando en cada estado conformacional y la función que representa la conformación en un momento temporal), si bien todos ellos juegan el mismo papel en las diferentes teorizaciones: dar cuenta de los sistemas empíricos.

Esto trae a colación la distinción entre las aplicaciones exitosas de cada teoría, pues del conjunto de aplicaciones sólo hay una en la intersección entre ambas: la "ecuación de Michaelis-Menten", en el lenguaje michaeliano o "los sistemas michaelianos" en MWC, y por ello ninguna reemplaza a la otra. 
En tercer lugar, nuestra última hipótesis es que hay una teoría implícita de mayor grado de generalidad que las anteriores que explicaría tanto los sistemas descritos por la teoría alosterica como por la michaeliana. Es decir, poseería un enorme poder de "unificación" (Díez 2002) y por alguna razón ha pasado desapercibida.

El sustento de tal hipótesis lo constituye el hecho de que, conociendo la forma lógica de las leyes de ambas teorías y mediante el instrumental formal estructuralista, es factible arribar a una "ecuación general" que integre tanto los ligandos de ambas teorías como la posibilidad de cambio conformacional. Esto permitiría capturar (explicar) los sistemas aquí tratados (más otros no discutidos pero que combinan distintos tipos de inhibidores y moduladores).

Si formalmente no hay impedimentos para el desarrollo de tal ecuación y existen además sistemas científicamente relevantes y de interés por explicarse ipor qué no encontramos la teoría en la bibliografía?

Bajo esta hipótesis epistemológica emprendimos otra búsqueda para hallar algunas aproximaciones.

Las investigaciones de cristalografía de rayos X de Max Perutz, que resultó una confirmación para el modelo MWC, es una de ellas: la estructura de la oxihemoglobina (hemoglobina unida al oxígeno) no se describió directamente debido a las dificultades para cristalizarla. Lo que se cristalizó fue el complejo hemoglobina-monóxido: "al ser este compuesto un inhibidor competitivo de la unión de oxígeno, la estructura que resulta de su unión es idéntica a la de la oxihemoglobina” (Vera 2010, p. 227). Cuando experimentalmente se encontraron dos conformaciones distintas (la forma desoxigenada que se correspondería al estado $\mathrm{T}$ y la oxigenada al R) se usó el inhibidor competitivo que, como el oxígeno, se une a la forma $\mathrm{R}$, tal como habíamos sospechado (¿o predicho?) en nuestra reconstrucción. Pero la misma aparece asociada al modelo MWC.

Más aún, en la bibliografía se enfatiza que la explicación de estos fenómenos la provee MWC. Ejemplo de ello es la enzima (alostérica) ACTasa, que en presencia del inhibidor (competitivo) malato, es presentada bajo el título "Efectos de la inhibición competitiva", alertando al final de la sección, que "modelos especiales han sido diseñados para dar cuenta de esta clase de comportamiento" (Cantor \& Schimmel 1980, pp. 945-945), pero curiosamente le sigue la exposición del "Modelo MWC". Y, como demostramos, dicho modelo no puede dar cuenta ni de éste, ni del ibuprofeno ni del envenenamiento por monóxido.

Más allá de lo aquí explicitado no aparecen rastros de la teoría unificadora, aunque sospechamos cada vez más de su existencia. Por ello emprendimos una búsqueda indagando distinto material, ahora de "matemática biológica". Recién aquí encontramos algún candidato, como la "generalización de MWC” de Papova y Sel'kov del '75 (Kesse \& Ben-Tal 2011). Pero, aunque prometedora, no podemos confirmar que sea la teoría buscada.

\section{Consideraciones finales}

Como resultado de este trabajo, hemos: i) presentado una primera reconstrucción de la teoría de Michaelis-Menten, ii) demostramos que, pese a lo que diga la bibliografía del área, no hay entre las teorías una relación de reducción, iii) que la relación es de incomensurabilidad parcial, iv) que ninguna explica el caso del ibuprofeno, el envenenamiento por monóxido o la ACTasa en presencia de malato, v. que nuestra sospecha de que existe una teoría que sí los explica (junto a varios otros sistemas) parece bastante sustentada, aunque no "confirmada". Sin embargo, podemos precisar cómo se espera sea su estructura. A continuación, presentamos de manera informal parte de la reconstrucción de esa teoría y cómo incorpora esas explicaciones por especialización.

Esta teoría de carácter más general deberá presentar una ley general, la que llamaremos de MonodMichaelis-Menten (LGMMM), que reemplace la 3o ley general de TMWC (mientras las otras dos leyes de TMWC en esta nueva teoría se mantendrían inalteradas). Tal ley debería adoptar la siguiente forma: 


$$
\delta_{(t)}=\frac{\left[l_{0 t} \cdot\left(1+\varepsilon_{I \tau t}\right) \rho o /\left(1+\varepsilon_{A r t}\right) \rho o\right] \cdot \varepsilon_{s t t} \cdot\left(1+\varepsilon_{s t t}\right) \rho o-1+\varepsilon_{s t t} \cdot\left[\left(1+\varepsilon_{i^{\prime} t}\right)+\varepsilon_{s \tau t} \cdot\left(1+\varepsilon_{i^{\prime \prime \prime} t}\right)\right] \rho o-1}{\left[l_{0 t} \cdot\left(1+\varepsilon_{I \tau t}\right) \rho o /\left(1+\varepsilon_{A r t}\right) \rho o\right] \cdot\left(1+\varepsilon_{s t t}\right) \rho o+\left[\left(1+\varepsilon_{i^{\prime} t}\right)+\varepsilon_{s \tau t} \cdot\left(1+\varepsilon_{i^{\prime \prime \prime} t}\right)\right] \rho o}
$$

Ella establece que el grado de actividad biológica (para una población de proteínas con ligando sustrato, moduladores e inhibidores no-moduladores), en cada momento, coincide con la proporción de subunidades unidas a ligando (en ambos estados conformacionales) sobre el total de uniones posibles.

En nuestros sistemas a explicar encontramos siempre proteínas con más de una subunidad que presentan cooperatividad entre sí, es decir que son proteínas del tipo alostérica. La primera restricción que se introduce a las leyes generales para capturar este tipo de comportamiento de la población es que, en ausencia de ligando, hay menor cantidad de proteína en estado $\mathrm{T}$ que en estado $\mathrm{R}$ y que la afinidad por el sustrato es mucho mayor para el estado R que para $\mathrm{T}\left(\kappa_{S \tau} \ll<\kappa_{S r}\right)$. Bajo estas restricciones la actividad biológica (para proteínas con cooperatividad) adquiere la siguiente forma:

$$
\delta(t)=\varepsilon_{s \tau t}\left[\left(1+\varepsilon_{i t}{ }^{\prime}\right)+\varepsilon_{s r t}\left(1+\varepsilon_{i^{\prime} t}^{\prime \prime}\right)\right]^{\rho o-1} \div\left[\nu_{0 t \cdot}\left(1+\varepsilon_{I t t}\right)^{\rho o} /\left(1+\varepsilon_{A r t}\right)^{\rho o}\right]+\left[\left(1+\varepsilon_{i t}{ }^{\prime}\right)+\varepsilon_{s r t} \cdot\left(1+\varepsilon_{i}^{\prime \prime} t\right)\right]^{\rho o}
$$

Si se añade además que no hay presencia de moduladores alostéricos (ni activadores ni inhibidores) en el sistema $\left(\varepsilon_{A r t}=\varepsilon_{I t t}=0\right)$ y todas las subunidades de la proteína se pueden unir o al sustrato o a algún inhibidor no-modulador, la forma que adquiere ahora la ecuación de actividad biológica es:

$$
\delta_{(t)}=\varepsilon_{s t t^{\prime}} \cdot\left[\left(1+\varepsilon_{i t}{ }^{t}\right)+\varepsilon_{s r t}\left(1+\varepsilon_{i}^{\prime \prime} t\right)\right]^{\rho o-1} \div \nu_{0 t}+\left[\left(1+\varepsilon_{i t}{ }^{t}\right)+\varepsilon_{s r t} \cdot\left(1+\varepsilon_{i}^{\prime \prime} t\right)\right]^{\rho o}
$$

Por último, si agregamos a esta ley (que captura los sistemas alostéricos parecidos a los llamados "homotrópicos" del modelo MWC) la restricción de ausencia de inhibidor no competitivo $\left(\varepsilon_{i}{ }^{\prime}=0\right)$ y teniendo en cuenta que el inhibidor competitivo tiene afinidad por el estado conformacional $\mathrm{R}$ (como el sustrato), la actividad biológica toma el siguiente valor específico:

$$
\delta_{(t)}=\varepsilon_{s t} \cdot\left[\left(1+\varepsilon_{i t}\right)+\varepsilon_{s t}\right]^{\rho o-1} \div l_{0 t}+\left[\left(1+\varepsilon_{i t} t\right)+\varepsilon_{s r t}\right]^{\rho o}
$$

Así llegamos a una ley que no puede tener más restricciones que las introducidas (ley especial terminal) y sólo nos restaría instanciarla según las especificaciones de los casos de interés. Nótese que es la misma ley especial terminal la que captura los tres ejemplos vistos.

Finalmente, y en ausencia de una teoría general, vi) podemos precisar el porqué aparecen ambas teorías en los libros de textos especializados, reflejo de cómo se enseñan: los sistemas biológicos que explica una no los explica la otra, y viceversa. Son, por tanto, necesarias para capturar los sistemas biológicos relevantes de la bibliografía, aunque como también se demostró, no son suficientes.

Bibliografía

Alleva, K., Diez J.A. y L. Federico (2012), "La teoría MWC (Monod, Wyman y Changeux): El sistema alostérico”, Ágora 31(2): 225-250.

Balzer, W., Moulines, C.U. y J.D. Sneed (1987), An Architectonic for Science. The Structuralist Program, Dordrecht: Reidel.

Cantor, Ch.R. y P.R. Schimmel (1980), The Behavior of Biological Macromolecules (part III). New York: W.H. Freeman and Company.

Diez, J.A. (2002), "Explicación, unificación y subsunción teórica”, en González, W. (ed.), Pluralidad de la explicación cientifica, Barcelona: Ariel, pp. 73-93. 
Hempel, C.G. (1958), “The Theoretician’s Dilemma”, en Feigl, H., Scriven, M. y G. Maxwell (eds.), Concepts, Theories, and Mind-Body Problem, Minneapolis: University of Minnesota Press, pp. 37-98.

Jaramillo, J.M. (2012), “¿Es compatible la idea de inconmensurabilidad no trivial con la del progreso científico? Algunas razones de su compatibilidad”, en Lorenzano, P. y O. Nudler (eds.), El camino desde Kuhn: la inconmensurabilidad hoy, Madrid: Biblioteca Nueva, pp. 225-264.

Kesse, A. y N. Ben-Tal (2011), Introduction to Proteins: Structure, Function, and Motion, Chapman \& Hall/CRC Mathematical and Computational Biology Series, Boca Raton: CRC Press.

Lorenzano, P. (2012), "Estructura y explicaciones intencionales, inconmensurabilidad teórica y comparabilidad empírica en la historia de la genética clásica”, en Lorenzano, P. y O. Nudler (eds.), El camino desde Kuhn: la inconmensurabilidad hoy, Madrid: Biblioteca Nueva, pp. 289-350.

Monod, J.; Wyman, J. y J.P. Changeux (1965), “On the Nature of Allosteric Transitions: A Plausible Model”, Journal of Molecular Biology, 12: 88-118.

Vera, L.F. (2010), "La hemoglobina: una molécula prodigiosa”, Revista de la Real Academia de Ciencias Exactas Físicas y Naturales 104(1): 213-232.

WWPDB: Worldwide Protein Data Bank. Accesible en: https://www.wwpdb.org/ (último ingreso Marzo 2016).

Zou, H., Yuan, C., Dong, L., Sidhu, R.S., Hong, Y.H., Kuklev, D.V. y W.L Smith (2012), "Human Cyclooxygenase-1 Activity and its Responses to COX Inhibitors are Allosterically Regulated by Nonsubstrate Fatty Acids”, Journal of Lipid Research 53(7):1336-1347. 